\title{
An information system to manage the rollout of the antiretroviral treatment programme in the Free State
}

\author{
JE Kotzé, Ph.D. \\ Department of Computer Science and Informatics, University of the Free State \\ T McDonald, Ph.D. \\ Department of Computer Science and Informatics, University of the Free State
}

\section{Kev words}

HIV/AIDS, Antiretroviral Treatment Programme, Paper-based Information System, Palm Computer System, Hospital Information System, Data Warehouse

\section{Correspondence address}

Prof. Theo McDonald

Department of Computer Science and Informatics

University of the Free State

P.O. Box 339

Bloemfontein

9300

Tel:(051) 401-2297

Fax:(051) 401-2297

E-mail: theo@ufs.ac.za

\begin{abstract}
Curationis 33 (2): $60-68$
The Acquired Immune Deficiency Syndrome epidemic, caused by the Human Immunodeficiency Virus, is a global crisis which threatens development gains, economies, and societies. Within sub-Saharan Africa, where the epidemic began the earliest and the HIV prevalence is the highest, African countries have death rates not seen before. In South Africa the epidemic has a devastating impact which creates profound suffering on individuals and their families, and the impact on the socio-economic level is of great concern. The eradication of HIV/AIDS represents one of humanity's greatest challenges, which requires co-operation and comprehensive collaboration between many different role players. In this endeavour clinical information plays a major role.
\end{abstract}

To combat the effect of the disease, the Free State Department of Health started with the provisioning of antiretroviral therapy in the public health sector. The objective of this paper was to address the challenges they faced in order to develop and implement an information system to manage the rollout of antiretroviral treatment effectively. They started with a paper-based system to collect vital information. It was followed by a palm computer project that was initiated to electronically capture the data collected by the paper-based system. This system was then replaced by a comprehensive Hospital and Clinic Information System which was acquired and customised for the antiretroviral data collection process. Research partners developed a standalone antiretroviral data warehouse for collecting information associated with the monitoring and evaluation of the Free State antiretroviral and HIV/ AIDS treatment programme. The data warehouse successfully produced several management information reports to the antiretroviral management team. A need was identified to design a comprehensive antiretroviral data warehouse that will integrate data from several operational sources which are all associated with HIV/AIDS. 


\section{Introduction}

The Acquired Immune Deficiency Syndrome (AIDS) epidemic, caused by the Human Immunodeficiency Virus (HIV), is a global crisis which threatens development gains, economies, and societies. The epidemic has evolved in different ways in various parts of the world, and at varying speeds. In many regions it is still in its early stages. AIDS is unique in human history in its rapid spread, its extent and the depth of its impact. In its yearly Global Report on AIDS, the United Nations reported that in the 20 years of the disease's existence, almost 20 million people already died and 33 million people (range: 30.3 -36.1 million) worldwide are living with HIV (UNAIDS, 2008c:32).

Within sub-Saharan Africa, where the epidemic began the earliest and the HIV prevalence is the highest, African countries have death rates not experienced since the 1950's or 1960's. According to UNAIDS (2008a:5), sub-Saharan Africa remains the most heavily affected by HIV, accounting for $67 \%$ of all people living with HIV and for $72 \%$ of AIDS deaths in 2007. The UNAIDS Global Report estimated that the number of AIDS related deaths in South Africa in 2007 ranged anywhere between 270000 and 420000 (UNAIIS, 2008b:217).

In South Africa the epidemic has a devastating impact which creates profound suffering on individuals and their families, and the impact on the socio-economic level is of great concern. HIV/ AIDS is a major threat to the most productive segment of the labour force and contributes to reduced earnings, imposing huge costs on enterprises in all sectors through decreasing productivity, increasing labour costs, and loss of skills and experience. HIV/AIDS is affecting fundamental rights at work, with respect to workers and people living with and affected by HIV/AIDS. The epidemic and its impact strike hardest at vulnerable groups including women and children, therefore increasing existing gender inequalities and exacerbating the problem of child labour (UNAIDS, 2004:62).

The HIV/AIDS epidemic threatens the viability of health-care systems. Treating AIDS and related opportunistic infections are placing heavy burdens on the health-care system of South Africa and throughout the world. The eradication of HIV/AIDS represents one of humanity's greatest challenges, which requires co-operation, and comprehensive collaboration between science, governments, social institutions, the media, the professions, and the general public. In this endeavour information plays a major role.

In response to this epidemic the South African Government created the HIV/ AIDS and Sexually Transmitted Disease (STD) Strategic Plan. The purpose of the plan is to provide a broad national framework around four priority areas: prevention; treatment, care and support; research, monitoring and evaluation; human and legal rights. In November 2003, , the South African government adopted the Operational Plan for Comprehensive HIV and AIDS Treatment and Care, which included the provision of antiretroviral (ARV) therapy in the public health sector (TshabalalaMsimang, 2003).

The objective of this paper was to solve the challenges that faced the Free State Department of Health (FSDOH) in order to obtain management information on the rollout of the ARV treatment programme in the Free State. The research methodology that was applied will be provided in the next section. That will be followed by a background on the ARV model of care framework used. Next the paper-based solution that was adopted to collect vital information will be discussed. A short overview will be provided on a palm-pilot project which was initiated to electronically capture data collected by the paper-based solution. The final part will cover the implementation of a clinic information system to manage the rollout of the antiretroviral treatment programme.

\section{Research methodology}

The research methodology used by this study was action research. Butler, Feller, Pope, Murphy \& Emerson (2006) noted that in action research projects, researchers collaborate with practitioners to solve practical problems while expanding scientific knowledge. Baskerville (1999) characterizes information system action research as an increased understanding of an imme- diate social situation, with emphasis on the complex and multivariate nature of this social setting in the information systems (IS) domain. It simultaneously assists in practical problem solving and expands scientific knowledge.

The action research description (Susman \& Evered, 1978) details a five phases, cyclical process. The approach first requires the establishment of a client-system infrastructure or research environment. Then, five identifiable phases are iterated: 1) diagnosing, (2) action planning, (3) action taking, (4) evaluating and (5) specifying learning. Baskerville (1999) provides an explanation of these components. The clientsystem infrastructure is the specification and agreement that constitutes the research environment and provides the conditions under which action and change may be specified. The client will be the FSDOH and the researcher will be the author of this paper. The research environment will be the Free State Province and will include staff working for the FSDOH at different levels of management.

Diagnosing corresponds to the identification of the primary problems that are causing the organization's desire for change. Action planning is the collaborative effort of the researcher and the client to identify organizational actions to relieve or improve the specified problems. Acting taking then implements the planned action in a collaborative manner between the researcher and the client. A collaborative evaluation of the implemented plan is done to determine if the changes had the desired outcome. Specifying learning is formally undertaken last, but is usually an ongoing activity.

Baskerville (1999) emphasizes the point that action research produces highly relevant research results, because it is grounded in practical action, aimed at solving an immediate problem while carefully informing theory.

The sections of the rest of the paper correspond to the five phases of the action research approach. The Background section corresponds to the diagnosis phase. The ARV Information System section corresponds to the action planning and action taking phase and the Discussion section to the 


\begin{tabular}{|c|c|}
\hline $\begin{array}{l}\text { ARV Assessment Service Points } \\
\text { (Primary Care Nurse Practitioners) }\end{array}$ & $\begin{array}{l}\text { ARV Treatment Service Points } \\
\text { (Doctors) }\end{array}$ \\
\hline 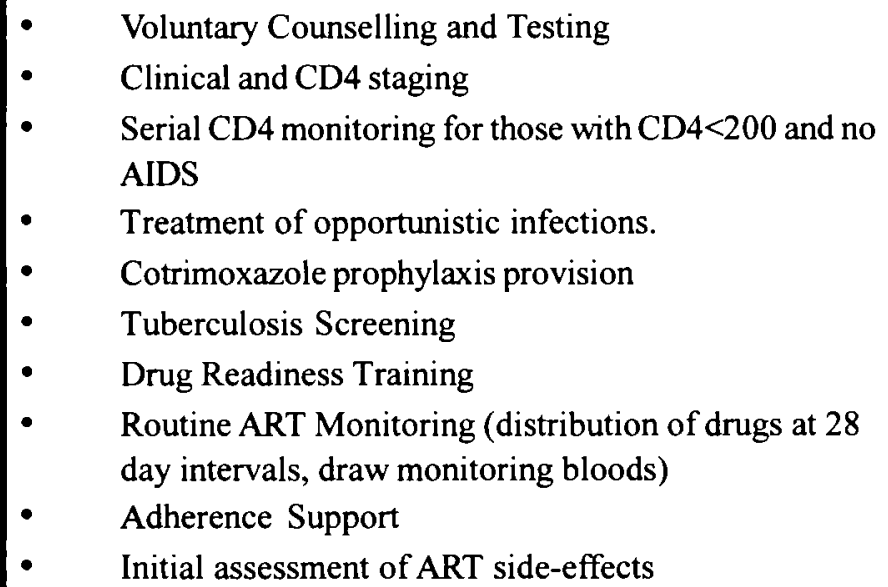 & $\begin{array}{l}\text { - ARV Baseline Assessments (including exclusion of } \\
\text { untreated tuberculosis, confirm eligibility for ARV treat- } \\
\text { ment) } \\
\text { Investigation to exclude undiagnosed opportunistic } \\
\text { infections (e.g. cryptococcal meningitis, } \\
\text { extrapulmonary tuberculosis) } \\
\text { Management of severe opportunistic infections prior } \\
\text { to ARV treatment initiation } \\
\text { Clinical review at planned ARV treatment initiation } \\
\text { - } \quad \text { Follow-up of patients on ARV treatment (review blood } \\
\text { results, decide on single drug or regimen changes) } \\
\text { Management of severe ARV treatment side-effects. }\end{array}$ \\
\hline
\end{tabular}

evaluation phase. The Conclusion section corresponds to the specifying learning phase.

\section{Background Information}

Following the South African National Policy (National Department of Health, 2003:1), the FSDOH established its Comprehensive Care, Management and Treatment of HIV and AIDS programme, which included the provision of highly active antiretroviral therapy (HAART) on the $3^{\text {rd }}$ May 2004. It was essential for the Free State Province that all levels of health care be involved from the beginning of the project in order to create a supportive environment which will include adults and children. With this, the hope would be that the ARV service would be as close as possible to the community to ensure greater access and an increase in treatment compliance. Taking this requirement into consideration, the Free State Province developed an initial framework model of care where doctor-led treatment sites were fed by nursedriven assessment sites from the surrounding area (Shai-Mhatu, 2006). The entire region's resources will, therefore, be used to help fight AIDS and support the ARV treatment campaign. It was important that the information system also make provision for this framework model of care which will be discussed in this paper.

Outlined below, was the proposed time frame for implementation of ARV treatment program in the Free State
(Chapman, 2003:5):

- $\quad$ Phase 1 (October 2003 - March 2004). Implementation at 3 ARV therapy units ( 1 regional hospital and 3 clinics each, 2 district hospitals and 3 clinics each).

- $\quad$ Phase 2 (April 2004 - March 2005). Implementation at 5 ARV therapy units ( 1 regional hospital and 3 clinics each, 4 district hospitals and 3 clinics each).

- $\quad$ Phase 3 (April 2005 - March 2008). Implementation at 16 additional ARV therapy units (16 district hospitals and 3 clinics each) and expansion to 100 clinics.

The Free State model of care (see Table 1) was structured around a nurse practitioner performing most of the treatment duties. In this model, clients or patients are screened (voluntary counselling and testing) at primary health care level (clinics) by nurse practitioners (called assessment service points). Those patients with a CD4 count below 200 or having AIDS already are referred to a nearby hospital for an initial assessment by a doctor (called treatment service points).

The doctor confirms whether the patient must go on ARV treatment. The patients are then referred back to the clinic for drug readiness training which usually lasts 3 weeks. On completion, they return to the hospital-based doctor for clinical review and initiation of treatment. Follow-up is primarily based at the clinic and nurses are responsible for 4 weekly distribution of medication dispensed by the hospital pharmacy, all blood monitoring (although results are reviewed by the doctor) and monitoring of adherence. Baseline assessment and 6 monthly follow-up assessments take place at the hospital by doctors who also issue prescriptions for ARV drugs.

\section{ARV Information System}

The ARV Information System evolved through several phases: a paper-based system, Palm hand-held computers, Hospital Information System, Clinic Information System and Data Warehouse. Each of these phases will now be discussed.

\section{Paper-based system}

The foundation of the ARV data collection process in the Free State was firstly built around the establishment of a formal paper-based information collection process. This was done to assist the users with three fundamental elements:

- Discipline to collect quality data

- Understanding of the ARV treatment programme

- Supplement the rollout of a Clinic Information System, once the users are familiar with the ARV treatment programme

Paper-based systems have long been 


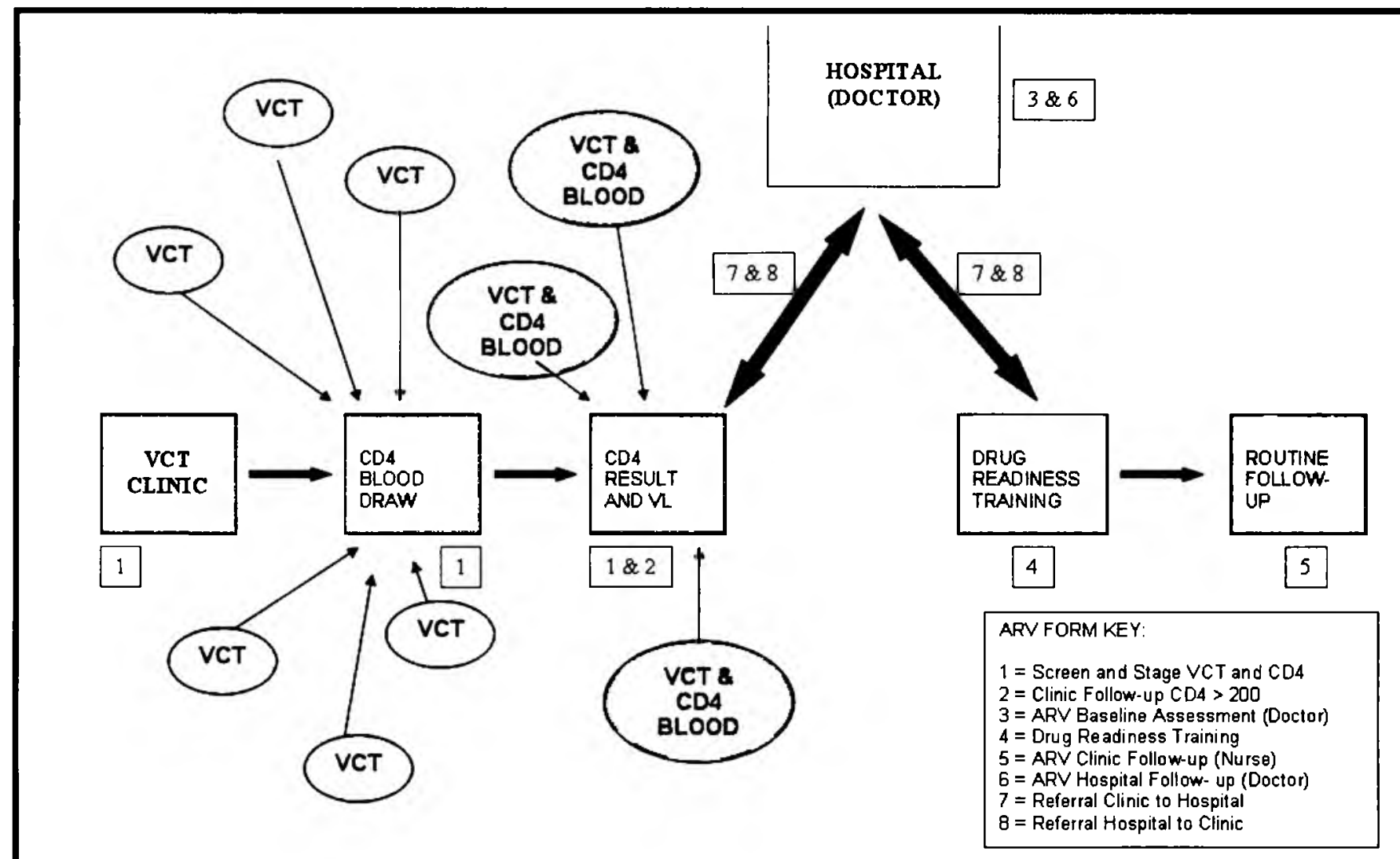

Figure 1: ARV Forms and Form Flow

the preferred medium for documenting clinical processes during consultations (Fairall, Botha, Bateman, Dong, et al., 2004:2). In many instances it is still the sole means of collecting data and compiling reports. For example in Malawi their AVR programme is monitored and evaluated by means of an ARV master patient card and an ARV patient register kept for each patient in the clinic (Lowrance, Filler, Makombe, Harries, et al., 2007:378). The FSDOH supported the continued use of the paper-based system, given that it would be risky to impose an alternative system (like direct electronic capture) on doctors and nurses dealing with the clinical aspects of a new and complex treatment programme. In Kenya a system was developed that transported the completed forms to a central site where data were electronically captured (Siika, Rotich, Simiyu, Kigotho, et al., 2005:347). For the FSDOH it was proposed that the paper-based collection of data be combined with early capture into an electronic system, preferably at the source (decentralized mobile data capture) where the data capturer is able to validate responses with the clinician who completed the form. This "editing" process had to be implemented right from the start of the programme to ensure that nurses and doctors understand how to complete the forms, and to encourage complete and accurate collection of data from the outset.

The following paragraphs will explain the contents and usage of each form:

\section{Voluntary Counselling and Testing (Screen and Stage)} Form

This form covers voluntary counselling and testing and CD4 staging. $\mathrm{Pa}$ tients may attend a clinic where voluntary counselling and testing (VCT) but not CD4 staging is offered. In this event the nurse could use the form as a referral to another clinic where CD4 staging is available. The form can also serve as a referral to the doctor at the local ARV treatment hospital. Referral criteria make provision for the referral of patients with CD4 counts $\geq 200$ with an AIDS-defining illness or on current or previous treatment.

\section{Clinic Follow-up: $C D 4 \geq 200$}

\section{Form}

This form records 6 monthly follow-up visits for patients with CD4 counts $\geq$ 200 , but not yet on ARV. It captures serial CD4 results. It has a strong focus on TB detection, and also makes provision for the documentation of cotrimoxazole prophylaxis in patients with late stage disease but CD $4 \geq 200$.

\section{ARV: Hospital Baseline Assessment Form}

This is the most detailed form and captures all the factors that may play a role in predicting treatment outcome. These factors include previous exposure to ARV, baseline CD4 and World Health Organization stage.

\section{Drug Readiness Training Form}

This form captures information on the three training sessions the patient attended at the assessment site.

\section{ARV: Clinic Follow-up Visit Form}

This form is designed to be completed once a month when patients return to 
receive treatment. It documents weight, current medication and screens for drug-related side effects and intolerance. It also retains a strong focus on the early detection of tuberculosis.

\section{ARV: Hospital Follow-up Visit Form}

This form is completed by a doctor at routine hospital follow-up visits. Initially these are planned at 3 month intervals, but frequency is likely to decrease as patients stabilize on treatment. The form makes provision for changes in opportunistic infection prophylaxis and ARV treatment.

\section{Referral: Clinic to Hospital Form}

Standardized and tailored referral forms could play a vital role in ensuring good co-ordination of care between clinic and hospital. This form makes provision for 5 common reasons for referral to a secondary level, and prompts the primary care clinician to provide information needed for appropriate clinical decision-making at hospital level.

\section{Referral: Hospital to Clinic Form}

This is a mirror version of the clinic to hospital referral form.

The different forms and the flow of the forms are shown in Figure 1.

\section{Medical Research Council (MRC) Palm Pilot Project}

Monitoring and evaluation are important activities of the public-sector ARV treatment programme. For this purpose structured records for collecting patient-specific information were developed. It was also envisaged that this will teach the future users of the Clinic Information System discipline in terms of data collection and data validation. As was highlighted in the previous section, in total eight paper forms have been designed and implemented at ARV assessment and treatment sites and hospitals throughout the Free State Province. All the forms are one A4 page in length, with the exception of the hospital baseline assessment form which is two pages long.

The MRC developed user defined screens on the Palm handheld compu- ter which were based on the paper forms. Seebregts, Zwarentein, Mathews, Fairall, et al. (2009) describe the development and evaluation of the Palm Pilot system for data collection in the Free State Department of Health. The data capturers at each assessment and treatment site capture information which was filled in on the paper forms onto the Palm Pilot handheld computer. At regular intervals, data was exported from the Palm Pilot handheld computer and uploaded to the MRC via a modem and telephone line situated at each clinic. This was an interim data collection system and planned to be discontinued once the Hospital and Clinic systems were online and operational at all ARV assessment and treatment sites in the Free State. By the end of October 2006 all the Palm Pilots were phased out and replaced by the following systems.

\section{Hospital Information System}

According to the National Health Information System for South Africa Committee (NHIS/SA, 2006:1) document, a Hospital Information System (HIS) is conceived, from the outset, as a system which is fundamentally common to all hospitals and primary health care (PHC) centres and that it is an integral and a major part of the National Health Information System of South Africa (NHIS/SA, 2006:1). The document also states that any Hospital Information System (HIS) used in South Africa, should have the following core National Health Care/Management Information System (NHC/MIS) functions:

- $\quad$ patient registration
a core, or a minimum data set,
patient record
- $\quad$ appointment scheduling
patient billing

Another key requirement is that the HIS should also be modular and that it should be completely developed and proven. The features and functionalities required in the NHC/MIS system vary from hospital to hospital, and certainly vary from one PHC centre to another, depending on its size and scope of service. Hence the Applications Software of the NHC/MIS should be modular and flexible so that only relevant modules are implemented in each hospital and PHC centre (NHIS/ SA, 2006:1). "Complete" means that the NHC/MIS should support the functional requirements as listed by NHIS/ SA (2006:1). "Proven" means that the system, in its entirety, is demonstrably operational in an existing environment and the NHC/HIS must not include any costs for 'applications development' or 'modifications' (NHIS/SA, 2006:1).

Simultaneously, a distributed Patient Database must be created and linked, via the Wide Area Network (WAN) to all the hospitals and Primary Health Care centres to both contribute to and use the database. The HIS should enable any of its features to be organised around a central database and must support different levels of access enabling restricted views of the centralised database.

The FSDOH honoured these requirements when it implemented a HIS at three of its larger hospitals in 1996. The implementation was expanded between 2004 and 2006 with the inclusion of two more regional hospitals. All the hospitals using the HIS were feeding data into a central Patient Database, which in turn provided the ability to compile a minimum patient record. Taking the existing infrastructure into account, a decision was taken by the FSDOH top management team, that the Clinic Information System (CIS) to be used for all ARV clinics must be fully developed and linked with the existing HIS.

\section{Clinic Information System}

In the previous section it was highlighted that one of the key requirements of a Hospital Information System is to provide all the hospitals and PHC centres the functionality to contribute and use the central patient database. Furthermore, according to the NHIS/SA document (NHIS/SA, 2006:1), for any Hospital Information System to be developed, installed and initiated in the major public hospitals, an appropriately scaled-down version of the same Hospital Information System must be implemented in all the smaller hospitals and PHC centres. It was therefore imperative for the FSDOH that the existing Hospital Information System (Meditech) already used in its three major hospitals, be scaled down and implemented as the electronic system 


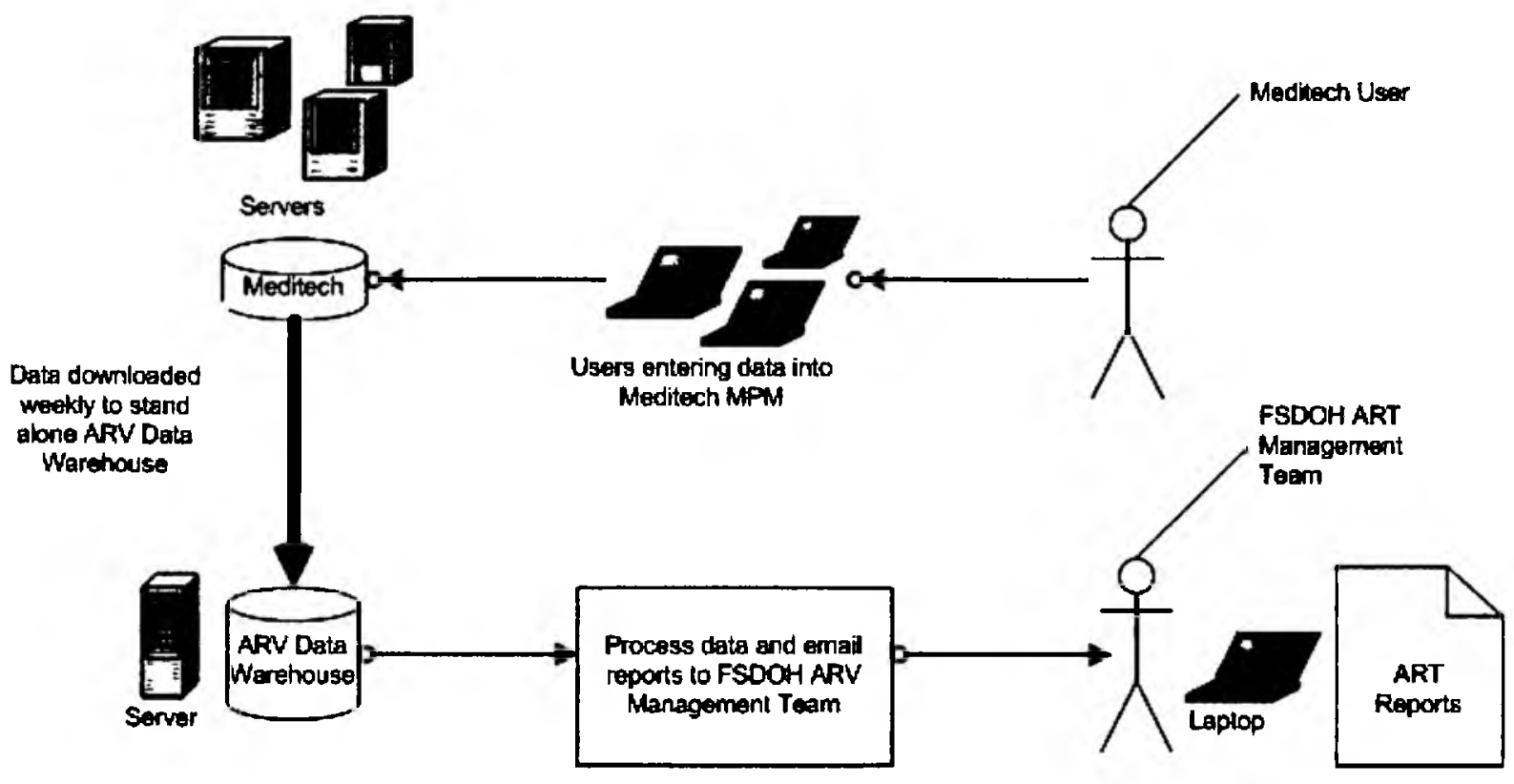

Figure 2: Model of the ARV Information System

for all the ARV PHC centres. For this paper, this system will hence be referred to as a Clinic Information System.

\section{Meditech}

While the paper-based information system was in use and data being captured by the interim Palm Pilot system, the acquisition and customization process for the Meditech software commenced. The initial investment of R2.5 million into the software licenses was justified by the fact that for sustainable long-term ARV monitoring and evaluation, the availability of electronic data available for analysis was pivotal. The customization process was not without its share of challenges and will be discussed later in this paper. The first pilot version was only ready in November 2004, while the ARV programme already commenced in May 2004. This meant that a backlog of paper forms which had to be captured while implementing the ARV sites at the same time.

Although the initial customization proc- ess delayed the implementation of all the Phase 1 ARV sites, the Department of Health was able to recover lost time and had most of the sites using a Clinic Information System by the end of May 2005.

The software that was customized and implemented is called Meditech Medical Practice Management Suite (Meditech MPM) and was customized to accommodate all the paper-based forms and the ARV treatment process that was already in place. This software, being one of the Meditech modules, is actually a scaled-down version of the larger Meditech Hospital Information System and comes complete with the following functionality:

- Community Wide Scheduling (CWS)

- Electronic Ambulatory Record (EAR)

- Authorization and Referral Management (ARM)

- Physician Desktop and Workload Management (PWM)
The following section will briefly explain each of the sub-modules of the Meditech MPM product suite to provide an overview of the system functionally and indicate how this will be used in the future for clinical information gathering.

\section{- Community Wide Scheduling}

(CWS): MPM manages appointment scheduling tasks by providing staff with booking options, check-in and registration tracking, links to patient profiles and conflict checking.

\section{- Electronic Ambulatory Record}

$(E A R)$ : Electronic patient records enable nurses at the clinics to track important patient data, such as medications, laboratory results, referrals, progress notes and patient history. EAR offers a flexible data entry screen, which does not require a huge investment in training time, or a lengthening of the encounter process. EAR follows the already developed paper-based workflow patterns which make sense for the physicians and nurses in either an ARV clinic or ARV hospital. The EAR module consists of the following health 
care sections: chronic and acute medical problems, medications (ARV drugs included), allergies and immunizations, CD4 count and viral load (Customer Defined Screen) and drug readiness training (Customer Defined Screen).

- Authorization and Referral Management (ARM): Integration of authorizations and referrals with registration, scheduling and recording of the patient's medical account are crucial. MPM offers integration with other MEDITECH applications and will contribute to the central Patient Master Index (PMI).

- Physician Desktop and Workload Management (PWM): MPM is equipped with a physicians/nurse desktop and workload management system which presents an interface to match a physician's/nurse's workflow. Additional fields were added to accommodate the ARV paper forms, which include drug readiness training, viral loads, CD4 counts and laboratory results.

\section{Medical Research Council Data Warehouse}

Whereas an operational database provides information on the day-to-day transactions, a data warehouse provides strategic information on historical data. The MRC in collaboration with the University of Cape Town (UCT) Lung Institute developed a standalone ARV data warehouse for collecting information associated with the monitoring and evaluation of the Free State ARV and HIV and AIDS Treatment Programme starting in November 2004. The data warehouse schema was based on the data received from the Palm Pilot handheld computers that were used at the Free State ARV assessment and treatment sites. The data was uploaded via modem to a staging database situated at the MRC and then loaded into the ARV data warehouse. Although the data warehouse produced several managerial reports to the FSDOHARV management team, its use was discontinued due to poor data quality which was associated with the Palm Pilot system. The Palm Pilot system was discontinued and replaced with the Meditech Hospital Information system which was implemented at ARV assessment and treatment sites. The existing data warehouse schema was adopted to accommodate the new operational data source database structure by the MRC in January 2006. A model of how this whole process functioned is shown in Figure 2.

By the end of 2006 the standalone data warehouse was handed over to the FSDOH to be incorporated into a proposed FSDOH data warehouse. The end product was to be a single "appropriately designed" ARV data warehouse made available for analysis to the relevant FSDOH managers involved with the ARV treatment programme.

\section{Discussion}

The customization and implementation process of the Meditech MPM software was not without a number of challenges. All of the challenges were identified at internal monthly management meetings where the customization and implementation of the NHC/HIS were discussed as a standing agenda point. Each of the listed challenges were carefully analyzed and solutions were proposed and implemented.

One of the first challenges faced was during the customization phase. Meditech South Africa had to develop customer-defined screens (CDS) to accommodate the already developed paper-based forms. With this came the notion of building an effective dataflow mechanism which follows the paperbased system in place, but also adhere to best-practice software engineering principles such as user friendliness, screen-to-screen serialization and ensuring data integrity and the avoidance of data duplication. This challenge took almost six months and led to the initial delay in the implementation of the Meditech software. A win-win solution was, however, proposed to accommodate the paper-based system in place, building on the user's familiarity with the complex ARV forms and data collection processes, and at the same time incorporating HIS functionality.

The second challenge was the issue of data quality. Because of the initial deployment of paper-based forms to both assessment and treatment sites in the province, numerous data elements of a patient were duplicated at each of the ARV PHC sites. With the implementation of the CIS, these duplicate data elements were identified as the data from the paper-forms was being captured into the system. For example, the same patient visited three different sites, resulting in three different patient files and three different patient numbers. Subsequently, as each site captured the backlog, the same patient was captured three times into the master patient index, each institution unaware of the other's action. The only viable solution was to merge these records on a weekly basis and to systematically remove the inconsistencies within the system. Users were also taught to make use of the master index lookup before creating a new electronic patient record. Another concept introduced to improve data quality was to implement the Meditech software in the entire district at the same time. This included the treatment sites and the referring assessment sites. With this method in place, all users were trained in the same week and also went live the same day. This also cultivated a culture of more efficient communication among the users in the same district.

The third challenge was implementing the Meditech MPM module itself. With the development of all the customerdefined-screens, the "out-of-the-box" functionality provided by MPM was overlooked. The implementation methodology purely focused on using the customer-defined-screens. During the pilot implementation phase at the Motheo district in November 2004, it became very clear that this approach will not work, because the MPM system functionality was now compromised. A strategy was devised to incorporate the "out-of-the-box" CWS functionality together with the EAR into all the customer-defined-screens. This new approach helped the assessment sites to schedule patient visits in advance and also to schedule their follow-up visit at the referral treatment site. This approach was adopted for all future site implementations and led to a reduction in capturing a patient's visit on the system.

The fourth challenge was to convince the FSDOH ARV Management team to include clinical staff (nurses and physicians) as users of the Meditech system. Most of the sites only had the data capturer and the admission clerk as users of the Meditech system. While the original idea was to create a longitudinal ARV patient record by capturing the paper-based information, it had 
little or no clinical value now, since the clinical staff was excluded to validate data quality. After lengthy discussions among all the provincial role-players it was eventually decided that the implementation process will also provide personal computers and printers for at least one nurse per site as well as Meditech access and training.

The fifth challenge was to provide a flexible state-of-the-art information system which gave the Department of Health a clear picture of the effectiveness of its rollout model, but at the same time adapt to ever changing needs. Free State was the first South African province to have a patient profiling system (Shai-Mhatu, 2006) and is still using the Clinic Information System at the time of writing this article. Numerous changes have been incorporated since its first implementation in 2004. Initially there were 8 adult forms but by the time of writing this article there were 28 forms, each with a corresponding CDS. Provision was also made to include data elements of children on the paper forms and the CIS. Since 2004, three phases of implementation were conducted. First phase was 27 ARV sites, second phase was 8 ARV sites and the third phase was 10 ARV sites. This meant by the time of writing this article, $45 \mathrm{ARV}$ sites were using the CIS in the Free State province.

Finally, approximately twenty (20) functional changes were made to the CIS to date. The most notable changes include screens capturing data elements on family planning, regimen changes, follow-up changes, patient staging changes, prophylaxis changes and user capture analysis. An ARV pharmacy module was also implemented. All of these functional changes were made to accommodate changing needs of doctors and professional nurses.

Although no formal evaluation was ever done on the CIS, it is clear from the active management involvement in solving the listed challenges, that the FSDOH are happy with the CIS as it continuously asks for functional changes, training and assistance from the vendor.

\section{Conclusion}

HIV/AIDS is a global crisis. To combat the effect of the disease, the South African government started with the provisioning of ARV therapy in the public health sector. To manage this process effectively in the Free State, an information system was implemented. The process started with a paper-based system to collect vital information. This was followed by a palmpilot project which was initiated to electronically capture the data collected by the paper-based system. This system was then replaced by a comprehensive Hospital and Clinic Information System which was acquired and customised for the ARV data collection process. Data was extracted from these systems and exported to a standalone ARV data warehouse located at the MRC. The purpose of this data warehouse was to collect information associated with the monitoring and evaluation of the Free State ARV and HIV and AIDS treatment programme. During the development and implementation of the information system several challenges were successfully overcame and the system has been in operation for a number of years. The logical next step now is to design and implement a single data warehouse for the FSDOH which will incorporate the data warehouse of the MRC. This data warehouse should also include data from other disparate operational systems which are closely linked to HIV/AIDS. Examples of such systems are: ARV Human Resources, Tuberculosis, Notifiable Diseases, Blood Results, and Hospitalizations. Such a data warehouse will provide strategic integrated information to management so that the ARV treatment programme can be managed effectively.

\section{Acknowledgements}

This work was made possible through the assistance and support of the University of Cape Town Lung Institute and the Medical Research Council in South Africa.

\section{References}

BASKERVILLE, RL 1999: Investigating Information Systems with Action Research. Communications of the Association for Information Systems, 2(19). http://cais.isworld.org/articles/219/default.asp?View=pdf $\& \mathrm{x}=78 \& \mathrm{y}=17$ Accessed 17 September 2007.

BUTLER, T; FELLER, J; POPE, A; MURPHY, C \& EMERSON, B 2006:

An action research study on the design and development of core IT arti- facts for knowledge management systems. http://csrc.lse.ac.uk/asp/aspecis/ 20060044.pdf Accessed 8 October 2007.

CHAPMAN, RD 2003: Plan for Implementation of ARV's in the Free State Province. Bloemfontein: Free State Department of Health. (Unpublished internal report).

FAIRALL,L; BOTHA,S; BATEMAN, E; DONG K; CHAPMAN, R; KOTZE, E \& VAN DER BANK, L 2004: Free State Anti-Retroviral Treatment Programme: Collection and Analysis of Routine Data. Bloemfontein: Free State Department of Health. (Unpublished internal report).

LOWRANCE, D; FILLER, S; MAKOMBE, S; HARRIES, A; ABERLE-GRASSE, $\mathbf{J}$ HOCHGESANG, M \& LIBAMBA, E 2007: Assessment of a national monitoring and evaluation system for rapid expansion of antiretroviral treatment in Malawi. Tropical Medicine and International Health. 12(3): 377-381.

NATIONAL DEPARTMENT OF HEALTH (SOUTH AFRICA) 2003: Cabinet's decision on the operational plan for comprehensive care and treatment of people living with HIV and AIDS. http://www.doh.gov.za/ docs/pr/2003/pr1119.html. Accessed 16 July 2008.

NATIONAL HEALTH INFORMATION SYSTEM OF SOUTHAFRICA COMITTEE (NHIS/SA) 2006: National Health Care Management Information System of South Africa (NHC/MIS). http://www.doh.gov.za/nhis/docs/ nchmis.htm. Accessed 15 May 2006.

SHAI-MHATU, P 2006: Fighting AIDS with Evidence. http://www.idrc.ca/en/ ev-101161-201-1-DO_TOPIC.html. Accessed 23 June 2010.

SEEBREGTS, CJ;ZWARENTEIN, M; MATHEWS, C; FAIRALL, L; FLISHER, AJ; SEEBREGTSs, C; MUKOMA, W \& KLEPP, K 2009: Handheld computers for survey and trial data collection in resource-poor settings: Development and evaluation of PDACT, a PalmTM Pilot interviewing system. To appear in the International Journal of Medical Informatics in 2009 
SIIKA,AM; ROTICH,JK; SIMIYA, CJ; KIGOTHO, EM; SMITH, FE; SIDLE, JE; WOOLS-KALOUSTIAN, K; KIMAIYO, SN; NYANDIKO, WM; HANNAH, TJ \& TIERNEY, WM 2005: An electronic medical record system for ambulatory care of HIV-infected patients in Kenya. International Joumal ofMedical Informatics. 74: 345-355.

SUSMAN, G\& EVERED, R 1978: An Assessment of the Scientific Merits of Action Research. Administrative Science Ouarterly. 23(4), 582-603.

TSHABALALA-MSIMANG, M 2003: Statement of Cabinet on a Plan for Comprehensive Treatment and Care for HIV and Aids in South Africa. http:// www.info.gov.za/speeches/2003/ $03111916531001 . h t m$. Accessed 23 June 2010.

UNAIDS 2004: Report on Global AIDS epidemic. http://www.unaids.org/ bangkok2004/report_pdf.html. Accessed 1 February $200 \overline{6}$.

UNAIDS 2008a: Report on the global AIDS epidemic 2008 - Executive summary.

http://www.unaids/org/en/ KnowledgeCenter/HIVData/ GlobalReport/2008/. Accessed 30 October 2008.

UNAIDS 2008b: HIV and AIDS Estimates and data, 2007 and 2001. Annexure 1.

http://data.unaids.org/pub/ G loba 1 Re port / $2008 /$ jcl510_2008_global_report pp211_234_enptf Accessed 30 October 2008.

UNAIDS 2008c: Status of the global HIV epidemic. Annexure 2. http:// whqlibdoc.who.int/unaids/2008/ 9789291737116_eng_Chapter2A.pdf. Accessed 30 October 2008. 